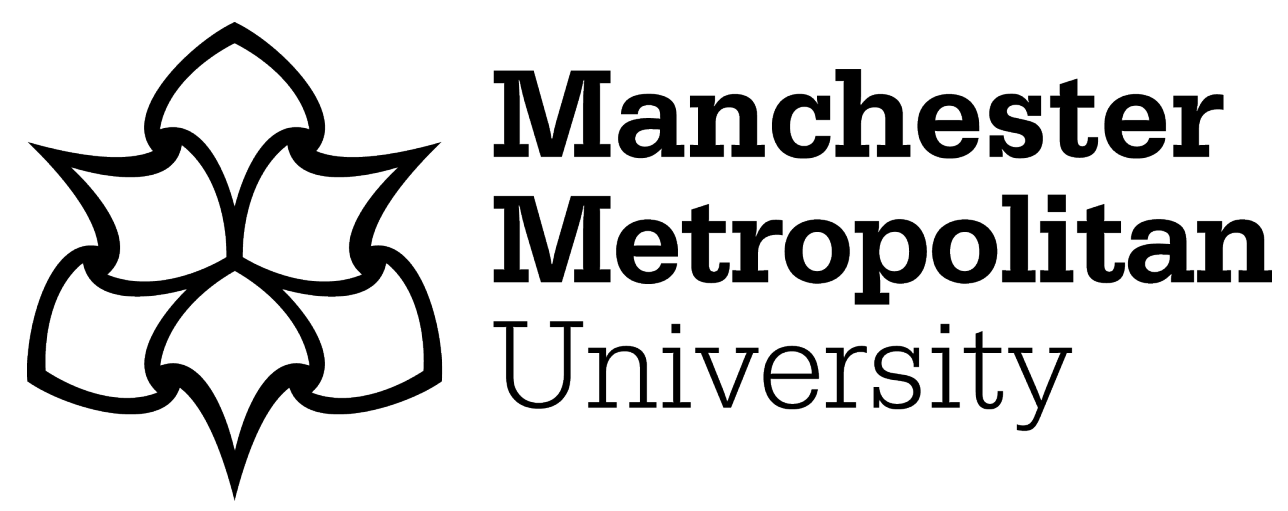

Comfort, P, Dos'Santos, T ORCID logoORCID: https://orcid.org/0000-00032715-0116, Jones, PA, McMahon, JJ, Suchomel, TJ, Bazyler, C and Stone, $\mathrm{MH}$ (2020) Normalization of early isometric force production as a percentage of peak force during multijoint isometric assessment. International Journal of Sports Physiology and Performance, 15 (4). pp. 478-482. ISSN 1555-0265

Downloaded from: https://e-space.mmu.ac.uk/626031/

Version: Accepted Version

Publisher: Human Kinetics

DOI: https://doi.org/10.1123/ijspp.2019-0217

Please cite the published version 
See discussions, stats, and author profiles for this publication at: https://www.researchgate.net/publication/336366155

\section{Normalization of Early Isometric Force Production as a Percentage of Peak Force During Multijoint Isometric Assessment}

Article in International journal of sports physiology and performance · October 2019 DOI: 10.1123/ijspp.2019-0217

\section{CITATIONS}

7 authors, including:

Paul Comfort

University of Salford

215 PUBLICATIONS 2,264 CITATIONS

SEE PROFILE

6 Paul A Jones

University of Salford

119 PUBLICATIONS 1,351 CITATIONS

SEE PROFILE

Some of the authors of this publication are also working on these related projects:

Biomechanical Assessment of Weightlifting Derivatives View project

Weightlifting overhead pressing derivatives View project
READS

272

Thomas Dos'Santos

Manchester Metropolitan University

66 PUBLICATIONS 508 CITATIONS

SEE PROFILE

John J McMahon

University of Salford

99 PUBLICATIONS 651 CITATIONS

SEE PROFILE 
1 Normalisation of early isometric force production as a percentage of peak force, during

4 Paul Comfort ${ }^{1,2, \#, \text { Thomas Dos'Santos }}{ }^{1}$, Paul. A. Jones ${ }^{1}$, John. J. McMahon ${ }^{1}$, Timothy. J.

$5 \quad$ Suchomel $^{1,3}$. Caleb Bazyler ${ }^{4}$, Michael, H. Stone ${ }^{4}$.

$6 \quad$ \#Corresponding author: Dr Paul Comfort

$7 \quad$ E-mail: p.comfort@salford.ac.uk

8 Twitter: @PaulComfort1975

9 ORCID ID: https://orcid.org/0000-0002-1131-8626

${ }^{1}$ Directorate of Sport, Exercise and Physiotherapy, University of Salford, Salford, Greater 13 Manchester, UK.

$14{ }^{2}$ Institute for Sport, Physical Activity and Leisure, Carnegie School of Sport, Leeds Beckett 15 University. Leeds. UK.

$16{ }^{3}$ Department of Human Movement Sciences, Carroll University, Waukesha, WI, USA

$17{ }^{4}$ Centre of Excellence for Sport Science and Coach Education, Department of Exercise and 18 Sport Science, East Tennessee State University, Johnson City, TN, USA

Preferred Running Heading: Normalisation of early force production

Submission Type: Original investigation

Abstract word count: 244

Word count: 2956

Figures: 1 


\section{Abstract}

Purpose: To determine the reliability of early force production (50-, 100-, 150-, 200-, $250 \mathrm{~ms})$ relative to peak force (PF) during an isometric mid-thigh pull (IMTP) and assess the relationships between these variables. Methods: Male collegiate athletes $(\mathrm{n}=29$; age: $21.1 \pm$ 2.9 years; height: $1.71 \pm 0.07 \mathrm{~m}$; body mass: $71.3 \pm 13.6 \mathrm{~kg}$ ) performed IMTPs during two separate testing sessions. Net PF and net force produced at each epoch were calculated. Withinand between-session reliability were determined by using intraclass correlation coefficients (ICC) and coefficient of variation (CV\%). Additionally, Pearson's correlation coefficients and coefficient of determination, were calculated to examine the relationships between PF and time-specific force production. Results: Net PF and time-specific force demonstrated very high to almost perfect reliability both within- and between-sessions (ICCs $0.82-0.97$; CV\% 0.35 $1.23 \%$ ). Similarly, time-specific force expressed as a percentage of PF demonstrated very high to almost perfect reliability both within- and between-sessions (ICCs $0.76-0.86$; CV\% 0.322.51\%). Strong to nearly perfect relationships $(r=0.615-0.881)$ exist between net PF and timespecific net force, with relationships improving over longer epochs. Conclusion: Based on the smallest detectable difference, a change in force at $50 \mathrm{~ms}$ expressed relative to $\mathrm{PF}>10 \%$ and early force production (100-, 150-, 200- and $250 \mathrm{~ms}$ ) expressed relative to $\mathrm{PF}$ of $>2 \%$ should be considered meaningful. Expressing early force production as a percentage of PF is reliable and may provide greater insight into the adaptations to the previous training phase than PF alone.

Key Words: Isometric mid-thigh pull; rapid force production; strength; monitoring 


\section{Introduction}

Maximal strength has been reported to be important for, and strongly associated with, performance in athletic tasks. ${ }^{1,2}$ Moreover, increases in strength have been shown to result in increases in athletic performance. ${ }^{2,3}$ Researchers have also reported strong associations between dynamic strength and maximal isometric force production., ${ }^{4,5}$ In addition to demonstrating the importance of a high maximal force capacity (i.e. a high maximal force production), the ability to rapidly produce high levels of force is paramount during athletic tasks, since there is a limited duration for the application of force during such activities. ${ }^{2,3}$ For example, during high velocity sprinting, foot contact times can be much less than $250 \mathrm{~ms}$, with a progressive decline in contact time as running velocity increases. ${ }^{6,7}$

Interestingly, there is a strong association between isometric peak force (PF) and isometric rate of force development (RFD) during single joint knee extension ${ }^{8,9}$ and during the isometric mid-thigh pull (IMTP). ${ }^{10,11}$ Additionally, these associations are stronger when RFD is calculated over longer epochs (for example $\mathrm{r}=0.57$ at $30 \mathrm{~ms}$ [from onset of force production] compared to $\mathrm{r}=0.89$ at $200 \mathrm{~ms}) .{ }^{9}$ Similarly, and as would be expected, force at specific timepoints (e.g. 20-, 40-, 60-... $200 \mathrm{~ms}$ ) are also closely related to PF during single joint knee extension. ${ }^{9,12}$ In addition, force at 100-, 150-, 200-, $250 \mathrm{~ms}$ has been reported to be associated with PF during a multi-joint isometric mid-thigh pull, ${ }^{1}$ with correlations between force at specific time points (50-, 90-, $250 \mathrm{~ms})$ and jump performance improving with an increase in duration (later time-points). ${ }^{11}$ It is important to reliably measure such changes in force production, to monitor the training status of athletes and inform future programming.

Aagaard et al. ${ }^{12}$ expressed isometric RFD across different epochs as a percentage of PF, during isometric knee extensions, to monitor adaptations to resistance training. Results revealed increases in early RFD (the first sixth of the time to PF), with the authors postulating that this 
may have been due to increases in motor neuron recruitment, motor unit firing frequency, myosin heavy chain isoform composition and sarcoplasmic reticulum calcium kinetics. Andersen et al. ${ }^{8}$ also demonstrated that early RFD (epochs from 0-10 ms up to 0-100 ms), expressed as a percentage of isometric PF indicates differential adaptations, in response to heavy resistance training, compared to later stage RFD (>200 ms). Similar observations for RFD expressed as a percentage of PF were also reported, with greater increases over longer epochs, although both early and late RFD were associated with isometric PF. The authors explained that a decrease in type IIx fibres may have negatively affected early RFD. Similar differential adaptations in early and late RFD were reported by Oliveira et al., ${ }^{13}$ with changes in RFD explained by the increases in isometric PF. Additionally, Blazevich et al. ${ }^{14}$ demonstrated that changes in early force production and early RFD are influenced by increases in fascicle length in response to training, which may partly explain these differential adaptations in early and late force development.

When comparing RFD normalised to isometric PF between athletes and controls, athletes demonstrated greater normalised RFD 0-50 ms, but not at other epochs. ${ }^{15}$ The authors attributed this to differences in neural activation and contractile properties of the muscle and tendon. As would be expected, absolute PF and RFD at all epochs were greater in athletes than controls. ${ }^{15}$ Similarly, Tillin et al. ${ }^{16}$ reported increases in force at specific time points (50-, 100-, $\left.150 \mathrm{~ms}\right)$, in response to four weeks of 'explosive' strength training, although, when normalised to isometric $\mathrm{PF}$, the only increase in force production occurred at $50 \mathrm{~ms}$, possibly due to enhanced agonist neural drive.

Isometric force at specific time-points has also been expressed in relation to $\mathrm{PF}$, in an attempt to explain training adaptations ${ }^{15,16}$ and differences between sexes, ${ }^{17}$ demonstrating that 
differences in early force production between sexes may be explained by differences in absolute force. While normalization of isometric force at specific time-points to PF during MVIC has been performed in single joint assessments, to the authors' knowledge, this approach has not been used in research using multi-joint isometric assessments of force. This is somewhat surprising as performance during single joint assessments of force do not appear to correlate well to athletic tasks. ${ }^{18,19}$ Multi-joint assessments, such as the IMTP demonstrate strong correlations with performance in a range of athletic tasks. ${ }^{20}$ Presenting data in this way may provide the coach with information explaining whether changes in early force production are in proportion to changes in $\mathrm{PF}$, therefore identifying if the athlete should emphasize maximal force production, or the ability to express force rapidly using appropriate training methods. The aim of this investigation was, therefore, to determine the reliability of force at specific time-points (50-, 100-, 150-, 200-, $250 \mathrm{~ms}$ ) expressed relative to PF, assessed during the IMTP. An additional aim was to identify if force at specific time-points are related to PF during the IMTP, as has been observed during single-joint assessments. ${ }^{9,21}$ It was hypothesized that force at specific time-points normalised to PF would be reliable and that PF and force at specific time-points would be associated, in line with previous research. ${ }^{1}$

\section{Methods}

\section{Subjects}

Male collegiate athletes from a variety of sports (rowing, field hockey, soccer) volunteered to participate in this investigation $(n=29$, age $21.1 \pm 2.9$ years; height $1.71 \pm 0.07 \mathrm{~m}$; body mass $71.3 \pm 13.6 \mathrm{~kg}$; power clean one repetition maximum $\left.1.12 \pm 0.09 \mathrm{~kg} \cdot \mathrm{kg}^{-1}\right)$. All subjects provided written informed consent, or parental assent as appropriate, and the study was approved by the university's institutional review board, in accordance with the Declaration of Helsinki. Subjects provided written informed consent and were all experienced in resistance training (resistance 
training age $2.1 \pm 0.6$ years) and familiar with the testing protocols, from previous performance of the IMTP used as a monitoring tool. Testing was completed at the start of the season, after a 4-week block of strength training.

\section{Design}

A repeated measures design was used, to determine the within- and between-session reliability of force at specific time points (50-, 100-, 150-, 200-, $250 \mathrm{~ms})$ normalised to PF during the IMTP, and identify relationships between time-specific force production and PF. Subjects were assessed twice (72 hours apart), to determine the reliability of the dependent variables. All testing occurred in-season, immediately after a one-week break from resistance training, after a previous four-week strength mesocycle. Testing was performed at the same time of day, with subjects asked to maintain their normal dietary intake and avoid strenuous exercise for at least 48 hours prior to testing.

Prior to testing, subjects performed a non-fatiguing standardised warm up consisting of body weight squats, forward and reverse lunges, and submaximal countermovement jumps. Further familiarisation and warm up trials were performed prior to the maximal IMTP, as described below.

\section{Methodology}

\section{Isometric Mid-thigh Pull}

Previously described procedures were adopted. ${ }^{20,22}$ An immovable cold rolled steel bar was positioned at a height that replicated the start of the second pull phase of the clean on a custom rack above a force platform. Once the bar height was established, the subjects stood on the force platform with their hands strapped to the bar, using standard lifting straps. ${ }^{1}$ Each subject 
adopted the posture that they would use for the start of the second pull phase of the clean, resulting in knee and hip angles of $139.5 \pm 3.3^{\circ}$ and $145.1 \pm 3.4^{\circ}$ respectively, in line with previous research. ${ }^{20,22}$ Individual joint angles were measured using a goniometer, recorded and standardised between testing sessions, in line with previous suggestions. ${ }^{20}$

Each participant performed three warm-up trials, one at $50 \%$, one at $75 \%$ and one at $90 \%$ of their perceived maximum effort, separated by one minute of rest. Once body position was stabilized (verified by watching the participant and the force-time record), the participants were given a countdown of " $3,2,1$, Pull". Any obvious pre-tension, determined as a force $>50 \mathrm{~N}$ above the subjects' system mass (body mass + bar mass), was not permitted prior to initiation of the pull. Subjects were instructed to pull against the bar "and push their feet into the ground as fast and hard as possible", which has previously been reported to produce optimal testing results. ${ }^{23}$ Each IMTP trial was performed for approximately five seconds, after at least one second of quiet standing in position prior to the start of the pull, ${ }^{20}$ and all participants were given strong verbal encouragement during each trial. Participants performed three maximal IMTP trials interspersed with two minutes of rest between trials. If PF during all trials did not fall within $250 \mathrm{~N}$ of each other, the trial was discounted and repeated after a further two minutes of rest, in line with previous recommendations. ${ }^{20,22}$

Vertical ground reaction force data were collected using a portable force platform sampling at $1000 \mathrm{~Hz}$ (Kistler Instuments, Winterthur, Switzerland), interfaced with a laptop computer and specialist software (Bioware 3.1, Kistler Instruments, Winterthur, Switzerland) that allows for direct measurement of force-time characteristics. Raw unfiltered, force-time data was exported for subsequent analysis in a bespoke Excel spreadsheet. 


\section{Data Analysis}

The onset of force production was defined as an increase in force that was greater than five standard deviations of force calculated during last 1 second immediately before the pull commenced. ${ }^{20}$ Body weight was subtracted from the original force-time curve to give the net force-time curve to prevent inflation of the associations between PF and time-specific variables which would occur if gross force was used. Net peak force was reported as the maximum force across the recorded net force-time curve. Subsequently, net force at 50-, 100-, 150-, 200- and $250 \mathrm{~ms}$ was identified. For between-session and correlational comparisons, the mean of the three trials was used.

\section{Statistical Analyses}

All statistical analyses were conducted in SPSS (version 26, IBM). Normality of all data was determined via Shapiro-Wilk's test, with all variables normally distributed $(p>0.05)$.

Reliability was assessed using two-way mixed model intraclass correlation coefficients (ICC) and $95 \%$ confidence intervals $(95 \% \mathrm{CI})$, with the 3,1 model used to determine within session reliability and the $3, \mathrm{k}$ model used between sessions. ${ }^{24}$ To determine the magnitude of the ICC, the values were interpreted as poor $(<0.50)$, moderate $(0.50-0.74)$, high $(0.75-0.90)$ and excellent $(>0.90) .{ }^{24}$ Coefficient of variation percentages $(\mathrm{CV} \%)$ were also calculated (standard deviation / mean $\mathrm{x} 100$ ) to determine the between session variability, with $<10 \%$ considered acceptable. ${ }^{25}$ The smallest detectable difference in change of the early force production, expressed as a percentage of $\mathrm{PF}$, was also calculated as follows: $(1.96 \times(\sqrt{ } 2) \times$ standard error of the mean [SEM]), with SEM calculated as: (SD [pooled] x $\sqrt{ }(1-\mathrm{ICC}))$. In addition, Hedge's $g$ effect sizes were calculated to determine if there were any meaningful differences between testing sessions and classified as trivial $(\leq 0.19)$, small $(0.20-0.59)$, moderate $(0.60-1.19)$, large $(1.20-1.99)$, and very large $(2.0-4.0){ }^{26}$ 
198 Pearson's correlation coefficients, with 95\% CI, and coefficient of determination $\left(\mathrm{R}^{2}\right)$, were 199 calculated to determine associations between PF and force at 50-, 100-, 150-, 200- and $250 \mathrm{ms,}$ 200 with the associated $p$ values adjusted using Bonferroni post-hoc correction for multiple 201 comparisons, and correlations interpreted as $<0.10,0.10-0.290 .30-0.49,0.50-0.69,0.7-0.89$ 202 and $\geq 0.90$ as trivial, small, moderate, large, very large and nearly perfect, respectively. ${ }^{26}$

204 Results

205 All force variables demonstrated good to excellent reliability both within- and between 206 sessions, with reliability tending to improve across longer time points. Coefficient of variation 207 values illustrated minimal variability $(<2.0 \%)$, while Hedge's $g$ effect sizes highlighted only 208 trivial differences between sessions (Table 1). 
210 Table 1: Within and between session reliability and variability of absolute isometric mid-thigh pull variables and time specific force variables

211 expressed as a percentage of peak force

\begin{tabular}{|c|c|c|c|c|c|c|c|c|}
\hline \multirow[b]{2}{*}{ Variable } & \multicolumn{2}{|c|}{ Session 1} & \multicolumn{2}{|c|}{ Session 2} & \multicolumn{4}{|c|}{ Between-Session Statistics } \\
\hline & Mean (SD) & ICC $(95 \% \mathrm{Cl})$ & Mean (SD) & ICC $(95 \% \mathrm{CI})$ & ICC $(95 \% \mathrm{Cl})$ & $\% \mathrm{CV}$ & $g$ & SDD \\
\hline Force $50 \mathrm{~ms}(\mathrm{~N})$ & $1069(237)$ & $0.827(0.705-0.910)$ & $1042(222)$ & $0.849(0.739-0.922)$ & $0.914(0.818-0.959)$ & 1.23 & 0.12 & $21.6(6.3 \%)$ \\
\hline Force $100 \mathrm{~ms}(\mathrm{~N})$ & $1276(346)$ & $0.914(0.845-0.957)$ & $1269(296)$ & $0.861(0.757-0.929)$ & $0.925(0.839-0.965)$ & 0.35 & 0.02 & $4.0(0.7 \%)$ \\
\hline Force $150 \mathrm{~ms}(\mathrm{~N})$ & $1537(453)$ & $0.938(0.880-0.967)$ & $1537(369)$ & $0.849(0.739-0.992)$ & $0.926(0.841-0.965)$ & 0.40 & 0.00 & $3.8(0.4 \%)$ \\
\hline Force $200 \mathrm{~ms}(\mathrm{~N})$ & $1765(494)$ & $0.933(0.878-0.967$ & $1745(407)$ & $0.849(0.737-0.922)$ & $0.929(0.849-0.967)$ & 0.44 & 0.04 & $9.9(0.95 \%)$ \\
\hline Force $250 \mathrm{~ms}(\mathrm{~N})$ & $1865(502)$ & $0.942(0.896-0.970)$ & $1840(447)$ & $0.894(0.816-0.945)$ & $0.954(0.903-0.978)$ & 0.70 & 0.05 & $11.7(1.02 \%)$ \\
\hline Peak Force $(\mathrm{N})$ & $2367(680)$ & $0.978(0.960-0.989)$ & $2390(674)$ & $0.965(0.934-0.983)$ & $0.977(0.951-0.989)$ & 0.72 & 0.03 & $6.9(0.4 \%)$ \\
\hline Force 50 ms (\%) & $46.3(7.4)$ & $0.764(0.608-0.875)$ & $44.7(6.8)$ & $0.724(0.551-0.851)$ & $0.821(0.622-0.916)$ & 2.51 & 0.22 & $0.020(9.6 \%)$ \\
\hline Force $100 \mathrm{~ms}(\%)$ & $57.9(8.9)$ & $0.786(0.642-0.887)$ & $54.4(8.9)$ & $0.724(0.551-0.851)$ & $0.831(0.639-0.921)$ & 0.68 & 0.39 & $0.005(1.5 \%)$ \\
\hline Force 150 ms (\%) & $66.0(10.9)$ & $0.797(0.658-0.893)$ & $65.7(10.8)$ & $0.722(0.546-0.850)$ & $0.866(0.713-0.937)$ & 0.32 & 0.03 & $0.003(0.5 \%)$ \\
\hline Force $200 \mathrm{~ms}(\%)$ & $75.7(8.7)$ & $0.834(0.714-0.914)$ & $74.4(9.8)$ & $0.858(0.732-0.931)$ & $0.815(0.607-0.913)$ & 1.19 & 0.14 & $0.011(1.7 \%)$ \\
\hline Force $250 \mathrm{~ms}(\%)$ & $79.6(8.2)$ & $0.786(0.648-0.884)$ & $77.9(7.7)$ & $0.813(0.654-0.906)$ & $0.801(0.581-0.906)$ & 1.53 & 0.21 & $0.013(1.9 \%)$ \\
\hline
\end{tabular}

$\mathrm{SD}=$ Standard Deviation; ICC = Intraclass Correlation Coefficient; $\mathrm{Cl}=$ Confidence Intervals; $\mathrm{CV}=$ Coefficient of Variation; $\mathrm{g}=$ Hedge's $g$ Effect Size; SDD = Smallest Detectable Difference 
213 Similarly, time-specific force variables expressed as a percentage of PF demonstrated high to

214 almost perfect reliability both within and between sessions. Coefficient of variation values

215 illustrated minimal variability $(<3.0 \%)$, while Hedge's $g$ effect sizes highlighted only trivial to

216 moderate differences between sessions (Table 1).

217 There were strong, significant correlations between force at each time-point and PF, with

218 progressive increases in the magnitude of the association from very strong to almost perfect,

219 as duration increased $\left(50 \mathrm{~ms}, \mathrm{r}=0.615[95 \% \mathrm{CI}=0.321-0.801], \mathrm{R}^{2}=0.378 ; 100 \mathrm{~ms}, \mathrm{r}=0.675\right.$

$220 \quad[95 \% \mathrm{CI}=0.410-0.835], \mathrm{R}^{2}=0.456 ; 150 \mathrm{~ms}, \mathrm{r}=0.720[95 \% \mathrm{CI}=0.480-0.860], \mathrm{R}^{2}=0.518$

$221200 \mathrm{~ms}, \mathrm{r}=0.796[95 \% \mathrm{CI}=0.606-0.900], \mathrm{R}^{2}=0.634 ; 250 \mathrm{~ms}, \mathrm{r}=0.881[95 \% \mathrm{CI}=0.760-$

$\left.2220.943], \mathrm{R}^{2}=0.0 .776 ; p<0.001\right)($ Figure 1). 

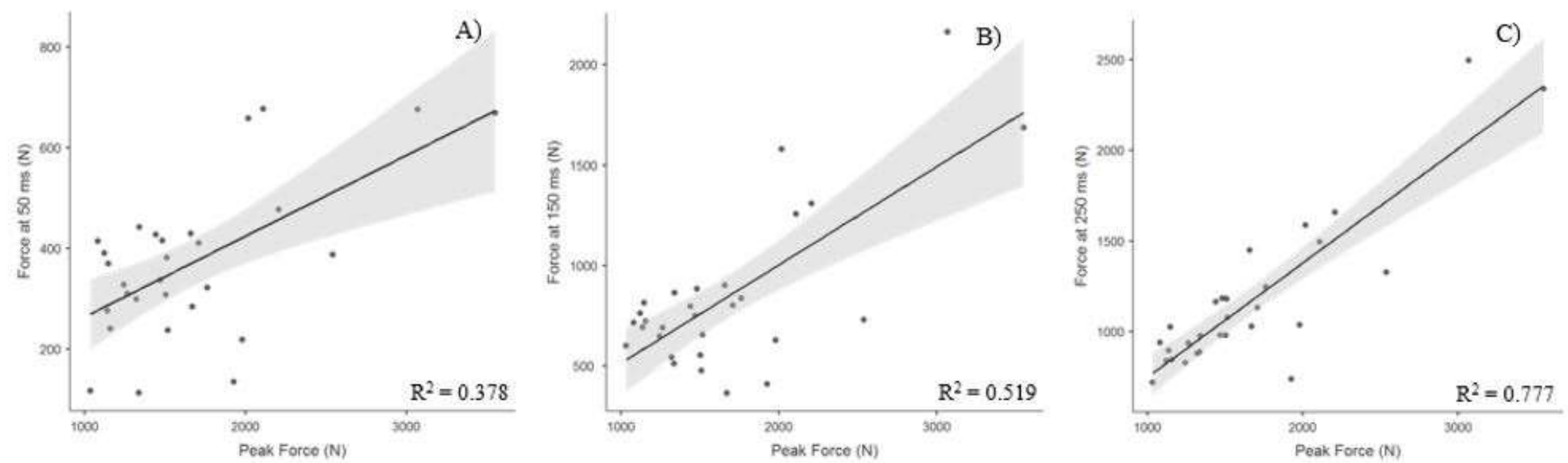

Figure 1: Associations between net force at A) $50 \mathrm{~ms}$, B) $150 \mathrm{~ms}$, C) $250 \mathrm{~ms}$ and net peak force. The shaded area represents the $95 \%$ confidence intervals 


\section{Discussion}

The aim of this investigation was to determine the reliability of net force at specific time-points (50-, 100-, 150-, 200-, $250 \mathrm{~ms})$ expressed relative to net PF, assessed during the IMTP, with the results highlighting good to excellent reliability within- and between sessions, with low variability and minimal differences between session, in line with the hypotheses. In addition, net force at each time-point was strongly associated with net PF, demonstrating coefficients of determination percentages of $38-78 \%$, which progressively increased with an increase in the duration over which net force was assessed.

The normalised time-specific net force values demonstrate lower reliability than the timespecific force values and net PF, as the process of normalizing these values combines the variability of both the time-specific net force and net PF. The normalised values, however, still exhibited high reliability both within- and between sessions, with low variability and minimal differences between sessions. Importantly, the smallest detectable difference of each of the normalised time-specific force values were $<10 \%$ and $<2 \%$ for normalised force from $100-$, $250 \mathrm{~ms}$.

When monitoring training adaptations, it would be useful to determine if any changes in early force production are in proportion to the change in net PF. For example, if an athlete's net PF increases and there is a disproportionately low change in early net force production (percentage of net PF decreases at specific time points), this may indicate that there is a deficit in rapid force production. In this scenario, the focus of the subsequent mesocycle should emphasize activities that focus on rapid force production rather than maximal force production. ${ }^{27,28}$ As such, this would ensure that the higher force generating capacity, developed during the previous mesocycle, can be appropriately expressed to enhance performance in sporting activities that require rapid force production, depending on the requirements of the athlete's sport. In contrast, 
252 if changes in early net force production are disproportionately greater than the change in net

253

254

255

256

257

258

259

260

261

262

263

264

265

266

267

268

269

270

271

272

PF (percentage of net PF increases at specific time points), it may be advantageous to emphasize maximal force production during the subsequent mesocycle. ${ }^{27,28}$ As such, expressing early net force production as a percentage of net PF should provide practitioners with additional insight regarding the development of their athlete and assist in the appropriate periodization of their training program.

It is worth noting however, that if net PF is low, indicating a low strength level, prioritizing maximal force production may be preferential, as strength development in weak individuals has been shown to be highly beneficial in terms of rapid force and power production. ${ }^{2,3,29,30}$ It is also worth noting, however, that during periods of high-volume training (e.g. hypertrophy) the associated residual fatigue is likely to result in an impaired ability to rapidly produce force. Additionally, rapid force production is more responsive to increases in training intensity compared to increases in volume. ${ }^{31}$

It is interesting that net PF explains $38-78 \%$ of variance in the force at specific time-points, and somewhat logical that a stronger association was observed as the time-point increased, with similar correlations previously reported in weightlifters. ${ }^{1}$ These findings highlight the importance of PF and therefore strength development, if the aim is to enhance an athlete's ability to express high forces rapidly. Such observations are in line with numerous studies using single joint assessment of force, ${ }^{9,21}$ the IMTP, ${ }^{1}$ and observations during dynamic tasks. ${ }^{2,3}$

Currently, the ideal percentage of net PF at different time points, along with how they relate to performance in athletic tasks, is yet to be identified. The authors, therefore, suggest that such thresholds be investigated in the future, along with the identification of the effect of different training intensities and volumes on the changes in early net force production relative to net PF, during the IMTP. Aagaard et al. ${ }^{12}$ previously postulated that increases in early force production 
are likely as a result of increases in motor neuron recruitment, motor unit firing frequency, myosin heavy chain isoform composition and sarcoplasmic reticulum calcium kinetics. Comparisons of changes in early force production and PF, as highlighted in this study, may permit identification of whether changes are as a result of more efficient force production, or as a result of increased PF.

\section{Practical Applications}

Coaches should consider changes in the percentage of net PF that can be produced during early time points (100-, 150-, 200- and $250 \mathrm{~ms})$ of $>2 \%$ as meaningful, whereas changes in net $\mathrm{PF}$ at $50 \mathrm{~ms}$, expressed as a percentage of net PF should be considered meaningful when $>10 \%$. Researchers and practitioners should determine the percentage net PF that can be produced during early time points, in addition to the absolute values, as this may provide greater insight into the adaptations to the previous phase of training and equip the practitioner with information regarding the requirements of the subsequent phase of training. If the percentage of net PF has increased for a given time point, additional emphasis on maximal force production (i.e. strength) may be warranted, while a decrease in percentage would indicate that additional emphasis should be placed on the development of rapid force production (See Haff and Nimphius ${ }^{32}$ for more detail regarding the application of such training principles). It is, however, important for coaches to also understand the effects that each training phase is likely to have on both PF and temporal aspects of force production, although further research should be conducted in this area. 
300

301

302

303

304

305

306

307

308

309

310

311

312

313

314

315

316

317

318

319

320

321

322

323

324

325

326

327

328

329

330

331

332

333

334

335

336

337

\section{Conclusions}

Expressing early net force production (50-, 100-, 150-, 200- and $250 \mathrm{~ms})$ as a percentage of net PF, during the IMTP, is reliable and may provide additional insight into the temporal aspects of force production.

Funding Details: No funding was received for this investigation

Disclosure Statement: The authors declare no conflict of interest or direct financial interest from the applications of this research

\section{References}

1. Beckham G, Mizuguchi S, Carter C, et al. Relationships of isometric mid-thigh pull variables to weightlifting performance. J Sports Med Phys Fitness 2013;53:573-81.

2. Suchomel TJ, Nimphius S, Stone MH. The Importance of Muscular Strength in Athletic Performance. Sports Med 2016;46:1419-49.

3. Suchomel TJ, Nimphius S, Bellon CR, Stone MH. The Importance of Muscular Strength: Training Considerations. Sports Med 2018;48:765-85.

4. McGuigan M, Winchester JB. The relationship between isometric and dynamic strength in collegiate football players. J Sports Sci Med 2008;7:101-5.

5. McGuigan MR, Newton MJ, Winchester JB, Nelson AG. Relationship between isometric and dynamic strength in recreationally trained men. J Strength Cond Res 2010;24:2570-3.

6. Weyand PG, Sternlight DB, Bellizzi MJ, Wright S. Faster top running speeds are achieved with greater ground forces not more rapid leg movements. J Appl Physiol 2000;89:1991-9.

7. Haugen T, Danielsen J, Alnes LO, McGhie D, Sandbakk O, Ettema G. On the Importance of "Front-Side Mechanics" in Athletics Sprinting. Int J Sports Physiol Perform 2017;E-pub ahead of print:1-24.

8. Andersen LL, Andersen JL, Zebis MK, Aagaard P. Early and late rate of force development: differential adaptive responses to resistance training? Scand J Med Sci Sports 2010;20:e162-9.

9. Andersen LL, Aagaard P. Influence of maximal muscle strength and intrinsic muscle contractile properties on contractile rate of force development. Eur J Appl Physiol 2006;96:46-52.

10. Haff GG, Carlock JM, Hartman MJ, et al. Force-time curve characteristics of dynamic and isometric muscle actions of elite women olympic weightlifters. J Strength Cond Res 2005;19:741-8. 11. Kraska JM, Ramsey MW, Haff GG, et al. Relationship between strength characteristics and unweighted and weighted vertical jump height. Int J Sports Physiol Perform 2009;4:461-73.

12. Aagaard P, Simonsen EB, Andersen JL, Magnusson P, Dyhre-Poulsen P. Increased rate of force development and neural drive of human skeletal muscle following resistance training. J Appl Physiol 2002;93:1318-26. 
13. Oliveira FB, Oliveira AS, Rizatto GF, Denadai BS. Resistance training for explosive and maximal strength: effects on early and late rate of force development. J Sports Sci Med 2013;12:4028.

14. Blazevich AJ, Cannavan D, Horne S, Coleman DR, Aagaard P. Changes in muscle force-length properties affect the early rise of force in vivo. Muscle \& Nerve 2009;39:512-20.

15. Tillin NA, Jimenez-Reyes P, Pain MTG, Folland JP. Neuromuscular Performance of Explosive Power Athletes versus Untrained Individuals. Med Sci Sports Exe 2010;42:781-90.

16. Tillin NA, Pain MTG, Folland JP. Short-term training for explosive strength causes neural and mechanical adaptations. Experimental Physiology 2012;97:630-41.

17. Hannah R, Minshull C, Buckthorpe MW, Folland JP. Explosive neuromuscular performance of males versus females. Experimental Physiology 2012;97:618-29.

18. Blackburn JR, Morrissey MC. The relationship between open and closed kinetic chain strength of the lower limb and jumping performance. J Orthop Sports Phys Ther 1998;27:430-5. 19. Ostenberg A, Roos E, Ekdahl C, Roos H. Isokinetic knee extensor strength and functional performance in healthy female soccer players. Scand J Med Sci Sports 1998;8:257-64.

20. Comfort P, Dos'Santos T, Beckham GK, Stone MH, Guppy SN, Haff GG. Standardization and methodological considerations for the Isometric Mid-Thigh Pull. Strength \& Conditioning Journal 2019;41:57-79.

21. Aagaard P, Simonsen EB, Trolle M, Bangsbo J, Klausen K. Effects of different strength training regimes on moment and power generation during dynamic knee extensions. European Journal of Applied Physiology and Occupational Physiology 1994;69:382-6.

22. Haff GG, Stone M, O'Bryant HS, et al. Force-Time Dependent Characteristics of Dynamic and Isometric Muscle Actions. J Strength Cond Res 1997;11:269-72.

23. Halperin I, Williams KJ, Martin DT, Chapman DW. The Effects of Attentional Focusing Instructions on Force Production During the Isometric Midthigh Pull. J Strength Cond Res 2016;30:919-23.

24. Koo TK, Li MY. A Guideline of Selecting and Reporting Intraclass Correlation Coefficients for Reliability Research. Journal of Chiropractic Medicine 2016;15:155-63.

25. Cormack SJ, Newton RU, McGuigan MR, Doyle TL. Reliability of measures obtained during single and repeated countermovement jumps. Int J Sports Physiol Perform 2008;3:131-44.

26. A scale of Magnitudes of Effect Statistics. 2002. (Accessed 01/01/18, 2018, at http://sportsci.org/resource/stats/index.html.)

27. Toji H, Kaneko M. Effect of multiple-load training on the force-velocity relationship. J Strength Cond Res 2004;18:792-5.

28. Harris GR, Stone M, O'Bryant HS, Proulx CM, Johnson R. Short-term performance effects of high power, high force or combined weight training methods. J Strength Cond Res 2000;14:14-20. 29. Cormie P, McGuigan MR, Newton RU. Developing Maximal Neuromuscular Power: Part 2 Training Considerations for Improving Maximal Power Production. Sports Med 2011;41:125-46 30. Cormie P, McGuigan MR, Newton RU. Influence of strength on magnitude and mechanisms of adaptation to power training. Med Sci Sports Exerc 2010;42:1566-81.

31. Mangine GT, Hoffman JR, Wang R, et al. Resistance training intensity and volume affect changes in rate of force development in resistance-trained men. Eur J Appl Physiol 2016;116:236774.

32. Haff GG, Nimphius S. Training Principles for Power. Strength \& Conditioning Journal 2012;34:2-12 
386 Table and Figure Legends:

387 Table 1: Within and between session reliability and variability of absolute isometric mid-thigh 388 pull variables and time specific force variables expressed as a percentage of peak force

390 Figure 1: Associations between net force at A) $50 \mathrm{~ms}$, B) $150 \mathrm{~ms}$, C) $250 \mathrm{~ms}$ and net peak 391 force. The shaded area represents the $95 \%$ confidence intervals 